\title{
Behaviour analysis for Web-mediated active learning
}

\author{
Claus Pahl \\ Dublin City University \\ School of Computing \\ Dublin 9 \\ Ireland \\ Email: Claus.Pahl@dcu.ie \\ Phone: $++353+17005620$ \\ Fax: $++353+17005442$
}

\begin{abstract}
Software-mediated learning requires adjustments in the teaching and learning process. In particular active learning facilitated through interactive learning software differs from traditional instructor-oriented, classroom-based teaching. We present behaviour analysis techniques for Web-mediated learning. Motivation, acceptance of the learning approach and technology, learning organisation and actual tool usage are aspects of behaviour that require different analysis techniques to be used. A combination of survey methods and Web usage mining techniques can provide accurate and comprehensive analysis results. These techniques allow us to evaluate active learning approaches implemented in form of Web tutorials.
\end{abstract}

Keywords: Interactive Tutorial; World-Wide Web; Courseware; Learning Behaviour; Formative Evaluation; Empirical Methods; Web Usage Mining.

\section{INTRODUCTION}

Since its inception, the Web has been widely and successfully used as a platform for teaching and learning. Technology-mediated teaching and learning, however, requires adjustments in the teaching and learning process for both instructors and students. The complexity of the symbiotic relationship between learning and instructional design on one hand, and technology and tool mediation on the other needs to be understood. Rose (1999) observes that the words 'interactive' and 'interactivity' proliferate in texts on educational computing, despite their apparent lack of denotative value. However, it seems to be understood widely that interactive instruction is learner-controlled, an opportunity for students to engage in active, hands-on exploration (Northrup, 2001). Interactive tools can enable active learning in a constructivist style if they create a representation of reality in which learning is relevant. According to Ravenscroft, Tait, and Hughes (1998), students integrate the use of computerbased learning resources into their study habits in an incremental fashion. Instructors need to carefully analyse the learning behaviour with new educational technologies in order to support new student learning processes through an incremental instructional design approach. 
The Web-mediated interactive tutorial system that we are going to analyse is part of an undergraduate course in computing. This tutorial allows students to construct programming knowledge and acquire programming skills in the database language SQL through engaging and interactive exercises based on meaningful problems, (Pahl, Barrett, \& Kenny, 2004). At the core of the tutorial is an interactive submission feature that allows students to execute programs and that gives feedback on those submissions. Engagement in the learning process is, according to Northrup (2001), a key objective in interactive instruction. In self-controlled environments, students actively construct meaning to determine how to proceed in the learning activity.

The goal of this investigation is the behaviour analysis of tool-mediated active learning. We demonstrate novel analysis techniques for the evaluation of learning behaviour in tool-mediated, interactive environments that combines classical survey-based techniques with Web usage mining technology. The motivation to analyse and evaluate the students' learning behaviour and learning processes is to gain an understanding of student learning in interactive learner-controlled environments. This is a prerequisite for the successful and effective implementation of instructional design for active learning and for the empirical evaluation of the implementations.

\section{THE INTERACTIVE TUTORIAL}

An interactive tutorial is a software tool that facilitates active learning in a guided learning process. Learners learn to solve problems in a dialogue with the tool. The interactive tutorial we analysed is part of an undergraduate courseware system for a database course, part of a computing degree, with online lectures, tutorials, and labs that is implemented using Web technologies and accessed through Web browsers and plug-ins and that supports active and autonomous learning, see Fig. 1. This environment is the target of our experimental and empirical study of learning behaviour.

Solutions to programming problems, which are presented as a guided tour through the material, can be submitted through a Web interface to a remote database server, which executes the input and replies with data from a database, or error messages (right-hand side of Fig. 1). Scaffolding in form of feedback, self-assessment functionality, and links to background material is available (bottom and left-hand side of Fig. 1). The tutorial prepares the student for coursework, such as lab tests and projects, and final exams. The courseware system aims at providing the student with a realistic learning context by integrating features and problems into a learning environment that are similar to tools and tasks that would be faced by a database engineer in a real development scenario. 


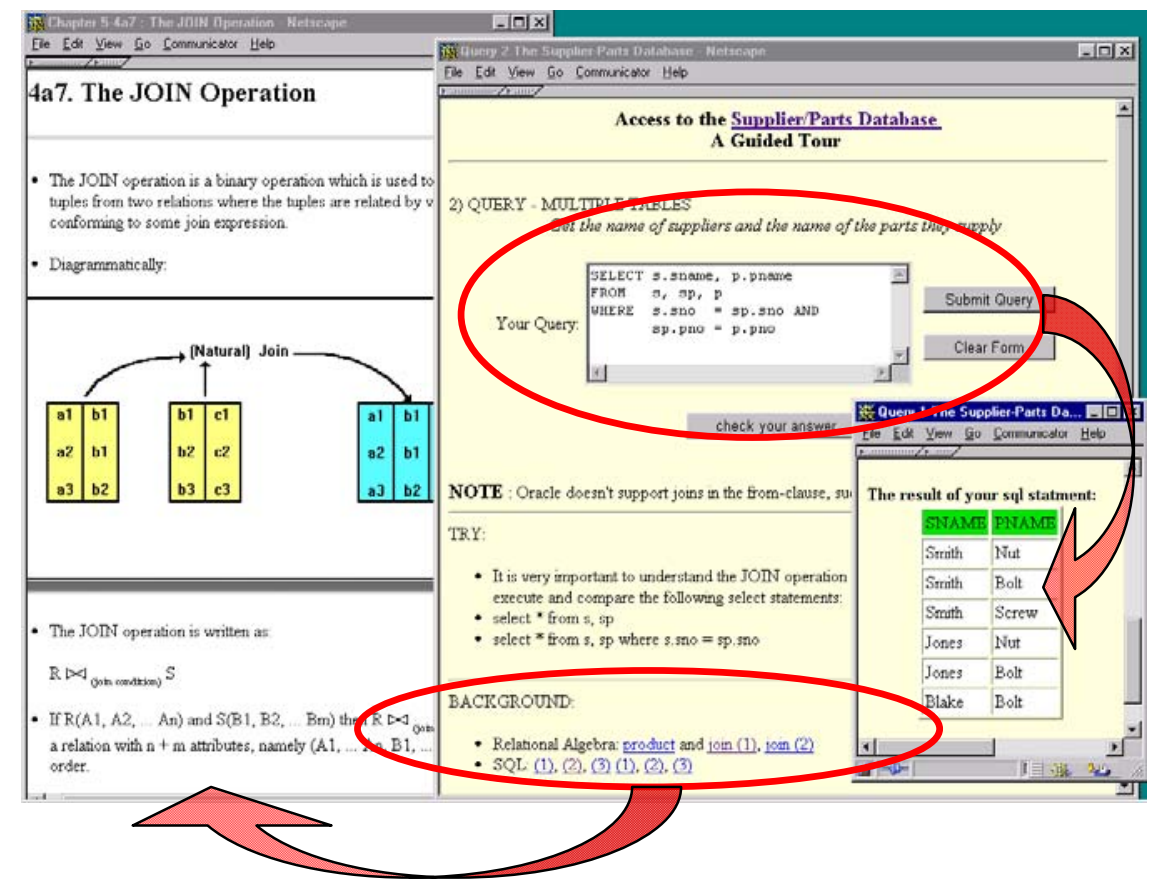

Figure 1. The interactive tutorial - with lecture material in the background.

\section{METHODS}

Our research goal is the analysis and evaluation of student learning behaviour in tool-mediated active learning environments. We define tool-mediated active learning as a software-supported approach to learning where a learner creates knowledge, i.e. a meaningful representation of some part of reality, within the software environment. Behaviour in learner-controlled environments is determined by the learners' motivation, their acceptance of pedagogical approach and technical environment, their learning organisation, and their activities in the environment (i.e. tool usage). Consequently, the instruments for the behaviour analysis include two instrument types: survey methods to address motivation and acceptance and Web usage mining techniques (Pahl, 2004) to capture organisation and usage in a Web environment. This combination provides a more complete and accurate picture than surveys and student observation alone (Kinshuk, Patel, \& Russell, 2000) or student tracking features available in various learning technology systems. We propose a novel, mainly quantitative method that combines classical survey methods with computational techniques for data mining and analysis. The survey design is addressed at the end of this and in the next section. We will focus here on mining techniques and the overall design of the analysis framework. 
Web mining is a technology that discovers and extracts knowledge from structured Web data - usually access logs that record requests from a Web browser. A Web log record - the basis for statistical analysis and data mining contains a user and/or machine ID, the time of the request, and the requested resource. To derive learning activities from navigation and interaction in Webbased systems is not always straightforward. Web logs record accesses to resources, which can be associated with activities.

Web mining has the advantage of being non-intrusive and useable at all times. Web log data can give a precise and objective account of student activities in Web-based systems. In addition to classical Web usage statistics such as number of hits in a period of time, Web usage mining allows a more targeted analysis of Web log data for educational purposes, (Zaiane \& Luo, 2001). Our analysis is based on two mining techniques developed for the educational context, (Pahl, 2004):

- Session classification. A Web log is a chronologically ordered list of Web requests. The first task is to identify learning sessions, which are defined as periods of uninterrupted usage of an individual user. The classification tries to identify purposes or activities of a session, for example interactive learning, attending a virtual lecture, or downloading resources.

- Behavioural pattern discovery. The Web log, if sorted by user, provides a sequential list of learner requests representing the learner activities in the system. The first task is to find sequential patterns (i.e. recurring sequences of requests). The second step is the identification of behavioural patterns such as repetition or the parallel use of features in these sequences and sequential patterns.

We have complemented a standard Web usage mining product with a research prototype for the education-specific features to implement Web usage mining. We recorded only information logged by standard Web servers - a fact that students were aware of and that should not have impacted their behaviour.

We have adopted complementary instruments - student surveys and observation-based Web usage mining - that allow us to address the different aspects of behaviour. Adding Web usage mining gives us an improved interpretative strength over classical methods for our behaviour analysis, as we have demonstrated in (Pahl, 2004). A benefit of the combination is the validation of behaviour-specific survey results and addition of preciseness through usage mining.

The behaviour of students in computer-based teaching and learning environments is influenced by the motivation to use the system and the acceptance of the approach. These two behaviour aspects relate concrete learning behaviour with the objectives and state-of-mind that have led to that behaviour. A learning activity is an engagement towards a learning objective. We distinguish two aspects of the student's concrete behaviour, which defines the learning activity. Firstly, the learning organisation addresses the study 
habits and captures how students organise their studies over a longer period of time. This includes how they plan to learn and work on coursework, and how they prepare for exams. Secondly, the usage of the system captures single learning activities and embraces how the student works with and behaves in the system in a single study session. Overall, we have identified four aspects of behaviour:

- Motivation - the reason to do something - causes the learner to act in some planned and organised way, giving the activities a purpose.

- Acceptance - to follow the learning approach and use the system willingly is crucial for the introduction of new educational technology.

- Organisation - the way the learning activities are planned and put into logical order - reflects the study habits and is guided by the purpose.

- Usage - the way the tool is actually used - reflects the actual learning activities.

Both the pedagogical approach and the Web-based system need to support the objectives that form the students' motivation in order to be accepted. The organisation is determined by the motivation - the objectives determine how activities are organised and executed. The usage follows the organisational plan to achieve the objectives. Motivation and acceptance are necessary to interpret organisation and usage. Except the motivation, we have analysed all aspects using both instruments for each category.

An iterative process of instructional Web design, based on a formative evaluation, facilitates feedback and exploration of new technologies. Formative evaluations are vital for identifying key design issues and for improving our understanding of pedagogical issues, (Kinshuk, Patel, \& Russell, 2000). Our analysis techniques combine mostly quantitative, but also qualitative aspects, leading to a more comprehensive picture of learning behaviour. This will result in a better understanding of how to develop new, effective types of learning environments.

We have surveyed and analysed the behaviour of two classes in two successive years with 79 and 112 students in each year, respectively. Of these 37 and 69, respectively, took part in the survey. Both classes were comparable with respect to age, sex, and also performance in coursework and exams. Both classes have used the tutorial system in the same version. Since no significant differences between the two classes emerged, their respective evaluation results shall not be distinguished. Tables 1, 2, and 3 below detail the questions and results of the questionnaire used in the student survey. All questions provided an open-ended part in order to record qualitative answers. Table 1 contains questions for which a number of alternative answers were given; the students were asked to rank these answers. Response categories for questions in Table 1 were decided based on standard categories from the literature and a pilot survey with open-ended questions. The questions in Tables 2 and 3 were presented in a Likert scale style - a 5-point scale (strongly disagree, disagree, undecided, agree, strongly agree - see Table 2) for a number of statements 
that students were asked to classify and a 5-point scale (traditional, rather traditional, undecided, rather virtual, virtual - see Table 3) where students were asked to compare delivery approaches. Table 4 presents session classification results. All reported results are statistically significant at the 95\% confidence level. Web mining was deployed constantly throughout the term.

\section{RESULTS}

\section{Motivation}

According to question Q1 - see Table 1 - there is a clear preference for practical course elements, i.e. coursework preparation, as the main motivation. A Web log analysis shows that the tutorial is mainly used during term to support coursework (about 2/3) and to a lesser extent (about 1/3) for the final exam preparation, which confirms the survey result. Question Q2 gives more insight into the motivation of the student's study organisation. From all alternatives offered in the survey, being 'always available' and 'self-paced learning' are the key advantages that students see in the system, i.e. these were ranked first (mean=1.63) and second (mean=2.22), respectively. Less than $4 \%$ of the students actually gave non-listed answers in the open-ended part.

Table 1. Student survey - motivation.

\begin{tabular}{|c|c|c|c|c|c|c|c|c|}
\hline \multirow[t]{4}{*}{ (Q1) } & \multicolumn{8}{|c|}{ For what purpose have you been using the interactive tutorial ? } \\
\hline & Answer & Count & Rank & $\left(1^{\text {st }}\right.$ & $\left.2^{\text {nd }}\right)$ & & & Mean \\
\hline & preparation for coursework & 98 & 1 & $71 \%$ & $21 \%$ & & & 1.13 \\
\hline & preparation for the exam & 94 & 2 & $29 \%$ & $67 \%$ & & & 1.63 \\
\hline \multirow[t]{6}{*}{$\overline{(Q 2)}$} & \multicolumn{8}{|c|}{ What were the main values of the interactive tutorial for you ? } \\
\hline & Answer & Count & Rank & $\left(1^{\text {st }}\right.$ & $2^{\text {nd }}$ & $3^{\text {rd }}$ & $\left.4^{\text {th }}\right)$ & Mean \\
\hline & always available & 92 & 1 & $62 \%$ & $22 \%$ & $10 \%$ & $6 \%$ & 1.63 \\
\hline & self-paced learning & 86 & 2 & $30 \%$ & $41 \%$ & $9 \%$ & $20 \%$ & 2.22 \\
\hline & easy Web access & 82 & 3 & $12 \%$ & $28 \%$ & $38 \%$ & $22 \%$ & 2.70 \\
\hline & integrated with lectures & 84 & 4 & $8 \%$ & $14 \%$ & $39 \%$ & $39 \%$ & 3.11 \\
\hline
\end{tabular}

\section{Acceptance}

Question S1 in Table 2 shows an overall acceptance of tool-mediated active learning as the pedagogical approach, which becomes even stronger when referring to the course with a strong practical element (database programming) in particular (Question S2). A positive attitude towards the approach usually goes hand in hand with frequent and regular usage - a correlation between these two variables confirms this. Comparing traditional and virtual tutorials see Table 3 - gives a more differentiated view on acceptance. Answers to question Q3 show no favourite - which demonstrates that students accept 
virtual tutorials as equally suitable and effective as traditional tutorials. We have asked the students about their preference of delivery mode with respect to performance in exams (Q4). The opinion is split. Nonetheless, this result shows the acceptance of virtual tutorials - virtual tutorials are at least as good as traditional ones - as a means to support one of the students' major objectives good coursework and exam performance. The answers to questions Q3 and Q4 demonstrate that, given an adequate online tool, virtual tutorials are feasible and they are accepted by learners as equally suitable and effective as traditional tutorials.

Table 2. Student survey - acceptance (S1,S2) and usage (S3).

\begin{tabular}{|c|c|c|c|c|c|c|}
\hline STATEMENT & Count & $\begin{array}{l}\text { strongly } \\
\text { agree }\end{array}$ & agree & $\begin{array}{l}\text { un- } \\
\text { decided }\end{array}$ & $\begin{array}{l}\text { dis- } \\
\text { agree }\end{array}$ & $\begin{array}{l}\text { strongly } \\
\text { disagre } \\
\mathrm{e}\end{array}$ \\
\hline $\begin{array}{l}\text { (S1) virtual courses are in } \\
\text { general } \\
\text { suitable for undergraduate } \\
\text { courses }\end{array}$ & 102 & $44 \%$ & $25 \%$ & $10 \%$ & $13 \%$ & $8 \%$ \\
\hline $\begin{array}{l}\text { (S2) having a virtual course as } \\
\text { part } \\
\text { of your degree programme is a } \\
\text { good idea }\end{array}$ & 102 & $53 \%$ & $25 \%$ & $9 \%$ & $9 \%$ & $4 \%$ \\
\hline $\begin{array}{l}\text { (S3) mixing the use of lectures } \\
\text { and } \\
\text { interactive tutorial is a good } \\
\text { idea }\end{array}$ & 101 & $49 \%$ & $33 \%$ & $10 \%$ & $7 \%$ & $1 \%$ \\
\hline
\end{tabular}

Another indicator for the acceptance of self-directed active learning is reflected by frequent and regular usage, in particular when alternatives are available. According to Web statistics students have worked in 19 sessions on average. About eight per cent of students have used the system twice or less. While Web mining shows that the tutorial system has not been used frequently and regularly over the whole term, it has, however, been used intensively in certain periods to fulfil a particular purpose.

\section{Organisation}

The organisation is reflected by the frequency and regularity of the usage. The access times in the Web log show high usage during later afternoon and early evening hours. The distribution over the week shows high usage in the middle of the week with $66 \%$ on Tuesdays and Wednesdays (weekly discussion meetings were held on these days), but also significant usage at weekends with close to $20 \%$. The study organisation overall - the self-paced learning aspect expressed through Q2 and Web mining results concerning frequency and 
regularity - shows a just-in-time learning approach with high usage immediately before coursework deadlines during the semester and before examinations.

Education-specific Web usage mining (Zaiane \& Luo, 2001; Pahl, 2004) can give us a clearer picture about the organisation than Web usage statistics. Session classification allows us to determine the purpose of sessions, for instance attending virtual lectures or practising in virtual tutorials, and to compare the session purposes of different periods. Table 4 shows percentages for the whole course system for two periods - the lecturing period during term and the exam preparation period following the lectures. A session can serve multiple purposes; thus, cumulative percentages can exceed $100 \%$. The purpose 'Organisational' includes downloading course notes and other material and look-up of course schedule and coursework results. 'Exploring' refers to an explorative behaviour, typical for the first sessions of a user. Surprising is the high number of organisational visits - even though these visits tend to be much shorter than lecture or tutorial sessions. Time series of session classifications allow us monitor the changing focus over time. We found dramatic changes in the classifications over time. Interactive services are heavily used during term, but less so for the exam preparation. Another change is the transition from a novice user with substantial explorative behaviour to an experienced one with more targeted behaviour that can be observed over time.

Table 3. Student survey - acceptance (comparison).

\begin{tabular}{lllllll}
\hline QUESTION & Count & traditional & $\begin{array}{l}\text { rather } \\
\text { traditional }\end{array}$ & $\begin{array}{l}\text { un- } \\
\text { decide } \\
\text { d }\end{array}$ & $\begin{array}{l}\text { rather } \\
\text { virtual }\end{array}$ & virtual \\
\hline $\begin{array}{l}\text { (Q6) in combination with } \\
\text { virtual lectures, would you } \\
\text { prefer tradit. or virtual } \\
\text { tutorials? }\end{array}$ & 100 & $30 \%$ & $13 \%$ & $13 \%$ & $18 \%$ & $25 \%$ \\
$\begin{array}{l}\text { (Q7) Do you think your exam } \\
\text { results would be better in a } \\
\text { traditional or a virtual } \\
\text { course? }\end{array}$ & & & & & & \\
\hline
\end{tabular}

\section{Usage}

Besides the long-term study organisation, analysing learning activities within a study session is crucial to understand how students learn. An abstract picture of the purpose(s) of each session is provided by session classifications, but we also need to look at how students interact with the system, whether they repeat units, or whether they combine interactive elements with lectures. A pattern analysis can answer these questions. 
Tut $_{1} ;\left[\text { LookUp }_{1} \mid \text { ExecQuery }_{1}\right]^{*} ;$ Tut $_{2} ;\left[\text { LookUp }_{2} \mid \text { ExecQuery }_{2}\right]^{*} ; \ldots$;

Tut $_{12} ;\left[\text { LookUp }_{12} \mid \text { ExecQuery }_{12}\right]^{*}$

This is a behavioural pattern describing a usage pattern for the interactive tutorial. The tutorial consists of 12 units - Tut $_{1}, \ldots$, Tut 12 - to be worked on sequentially, which is indicated through a semicolon (;). Within each unit students can iteratively $\left(^{*}\right)$ either look up background lecture resources (LookUp) or can execute an SQL query (ExecQuery) - options are separated by a vertical bar (|). A behavioural pattern analysis can extract such a pattern, and can, given a pattern, determine the overall support of the pattern by the class. For instance, $84 \%$ of all student sessions actually follow this pattern of mixing active tutorial learning and lecture look up - most of those sessions who do not are either very short or use a different order.

Question S3 (Table 2) shows that students recognise the potential of virtual courses to use tutorials and lectures at the same time, overcoming time and space constraints that apply to a traditional delivery. Question S3 gives an indication of the appreciation of this new style of learning mixing tutorials and lectures. This is confirmed by pattern analysis results, which show the proportion of students using the tutorial on its own or in combination with lecture resources. Lectures are usually used on their own. Interactive tutorials, however, are used to a large extent - Web mining confirms $77 \%$ - in combination with lecture resources. The students have looked up background material to solve specific problems interactively. While nearly all students avail of this feature in their first sessions, we observed a decrease of lecture usage during tutorials over time, indicating the increased knowledge, skills, and selfreliance of students.

Table 4. Web usage mining - session classification, in per cent (\%).

\begin{tabular}{llllll}
\hline $\begin{array}{l}\text { Activity } \\
\text { Period }\end{array}$ & Lectures & Tutorials & $\begin{array}{l}\text { Organisationa } \\
\text { l Exploring }\end{array}$ & Unclassified \\
\hline $\begin{array}{l}\text { Semester } \\
\text { Exam }\end{array}$ & $56 \%$ & $39 \%$ & $56 \%$ & $12 \%$ & $17 \%$ \\
\begin{tabular}{l} 
Preparation \\
\hline
\end{tabular} & $43 \%$ & $12 \%$ & $41 \%$ & $1 \%$ & $4 \%$ \\
\hline
\end{tabular}

\section{DISCUSSION}

Looking at the four behaviour aspects we found common expectations about motivation, essentially coursework and exam preparation, confirmed by the survey. Tool-mediated active learning is accepted as an equally effective means for learning - a positive result. The organisation analysis shows expected, but more undesirable results. In order to overcome the just-in-time approach to study organisation, the instructional design and course organisation would need 
to encourage a more regular use. The usage analysis provides again encouraging results. It shows that active and multi-modal learning are accepted and adopted. Overall, we have seen changing patterns, indicating both changes in the short-term focus of learning, but also in the long-term strategies that are used.

This behaviour evaluation shows the feasibility of tool-mediated active learning. Active learning can be supported by Web-based technologies. Students accept and use the system as a proper alternative to traditional forms of learning in particular for practical course elements. The analysis confirms steps we have already taken to facilitate tool-mediated active learning in a Web environment. The analysis shows that scaffolding techniques providing feedback, selfassessment functionality, and links to other services and background material including lectures, are as important as the learning activity itself within the interactive tutorial. One reason is that Web-based tutorials are used in a selfpaced and self-reliant way. For most of the students, our course was the first substantial exposure to tool-mediated learning. However, a substantial group had used similar systems before and we found evidence of a correlation between previous experience and high usage. This is an indicator for the change and refinement of learning strategies among students. Initially, students have used scaffolding and navigation support substantially, but over time a change towards self-reliance with respect to the content and also the usage of the tool was observed.

Important instructional design issues emerging from the analysis concern multimodal learning, feedback, and the learning organisation. Using the tutorial integrated with lecture resources can result in more problem-oriented multimodal learning, which organises different aspects such as theory and practice around a realistic problem. A wide awareness of this potential exists; however, a better implementation of this learning behaviour seems possible. Student responses, erratic behaviour, and some examples of repeated behavioural patterns show that individual feedback and scaffolding features are prerequisites to enable efficient and satisfactory usage of the system. Just-intime learning resulting is usage peaks is seen as undesirable with respect to knowledge retention. Weekly discussions that were introduced have helped to flatten these peaks and to encourage a more regular attendance.

\section{CONCLUSIONS}

The detection, analysis, and understanding of student learning processes in new forms of learning environments - such as tool-mediated active learning - is a prerequisite for the development of effective instructional design. The central problem is the adaptation of support for learning processes using new technologies. We have demonstrated the benefits of a learning behaviour analysis method based on combined survey and Web mining techniques that 
addresses the behaviour aspects motivation, acceptance, organisation, and usage. Although Web mining has limitations related to caching and other technical Web features, usage mining techniques enable constant, non-intrusive monitoring of student behaviour and the detection of behaviour changes, which supports the adoption of Web-based instructional techniques in an incremental process, (Coates \& Humphreys, 2001). This technique can be deployed for interactive Web-based tutorials where access logs reflecting learning activities are automatically generated.

Ravenscroft, Tait, and Hughes (1998) stress the importance of the appropriate level of student interaction with learning or knowledge media, referring to their experience with text-based and editable material for online lectures. Often, a distinction is made between educational content aimed at developing conceptual knowledge, problem solving, and analytical skills on one hand, and skills development, recognition, and memorisation on the other, (Weston \& Barker, 2001). The students' motivation in our case is the acquisition of skills, rather than knowledge, and good performance in practical coursework and examinations. Consequently, the form of interaction with course material supporting active learning of skills is different from knowledge-based learning. Other aspects such as a realistic setting for the interaction, for example in relation to project work or exam settings, become more important. Active learning provides this necessary type of interaction. Our conclusion - that the right level of interaction has to be designed and supported - is the same. The support of active learning through interactive tools needs to facilitate skills development in a realistic setting.

We found, based on our behaviour evaluations, that active self-controlled learning is an effective approach for practical, skills- rather than knowledgeoriented subjects. Interactive tools in a realistic setting that engage the students allow students to interact with the course content through its tool-based delivery medium in an adequate way. According to the students' opinion, tool-mediated active learning effectively replaces the instructor as a means for coursework and exam preparation to a large degree, in particular when direct contact with the instructor is not possible. The integration with other forms of learning provides an additional, beneficial context. However, using this technology, students are required to change their learning strategies. A constant analysis of student behaviour can help the instructor to support learning strategies and to accommodate changes in these strategies.

While we have analysed a computing course, Web technologies enable a wider range of subjects to be supported through active and dynamic Web pages, user-controlled animations, or submission, execution and feedback systems. These subjects need to aim at skills based on activities that involve some form of text processing or manipulation that is supported by Web technologies 


\section{REFERENCES}

Coates, D. \& Humphreys, B.R. (2001). Evaluation of Computer-Assisted Instruction in Principles of Economics. Educational Technology \& Society 4(2).

Kenny, C. and Pahl, C. (2005). Automated tutoring for a database skills training environment. ACM SIGCSE Symposium 2005. St. Louis, US. ACM Press. 2005.

Kinshuk, Patel, A. \& Russell, D. (2000). A Multi-institutional Evaluation of Intelligent Tutoring Tools in Numeric Disciplines. Educational Technology \& Science 3(4).

Xu, L., Pahl, C. \& Donnellan, D. (2003). An Evaluation Technique for Content Interaction in Web-based Teaching and Learning Environments. International Conference on Advanced Learning Technologies ICALT'2003. IEEE Press. 294285.

Northrup, P. (2001). A Framework for Designing Interactivity into Web-based Instruction. Educational Technology 41(2), 31-39.

Pahl, C. (2004). Data Mining Technology for the Evaluation of Learning Content Interaction. International Journal on E-Learning IJEL, 3(4), 48-59. AACE.

Pahl, C., Barrett, R., \& Kenny, C. (2004). Supporting Active Database Learning and Training through Interactive Multimedia. International Conference on Innovation and Technology in Computer Science Education ITiCSE'04. Leeds, United Kingdom. ACM.

Ravenscroft, A., Tait, K. \& Hughes, I. (1998). Beyond the Media: Knowledge Level Interaction and Guided Integration for CBL Systems. Computers and Education, 30 (1/2), 49-56.

Rose, E. (1999). Deconstructing Interactivity in Educational Computing. Educational Technology 39(1), 43-49.

Weston, T.J. \& Barker, L. (2001). Designing, Implementing, and Evaluating Web-Based Learning Modules for University Students. Educational Technology, 41(4), 15-22.

Zaiane, O.R. \& Luo, J. (2001). Towards Evaluating Learners' Behaviour in a Web-Based Distance Learning Environment. Proc. IEEE International Conference on Advanced Learning Technologies ICALT'01. 357-360. IEEE Computer Society. 